\title{
NONOSCILLATION CRITERIA FOR ELLIPTIC EQUATIONS IN CONICAL DOMAINS
}

\author{
W. ALLEGRETTO
}

\begin{abstract}
Nonoscillation criteria are established for a class of higher order elliptic equations defined in conical domains.
\end{abstract}

There has been considerable interest in recent years in establishing oscillation and nonoscillation criteria for elliptic equations. We refer the reader to the books by C. A. Swanson [7] and K. Kreith [4], where extensive bibliographies may be found.

While most of the interest has centered on second and fourth order equations, Allegretto [1] and Noussair and Yoshida [6] have recently established nonoscillation criteria for higher order equations. All of these results however, except for one of the criteria of [6], were obtained for the case where the unbounded domain considered was the complement of a sphere.

It is the purpose of this paper to establish some nonoscillation criteria for higher order elliptic equations which are defined in domains contained, at infinity, in cones of angle not greater than $\pi / 2$. Our criteria, unlike the result of [6] mentioned above, will depend both on the angle of the cone considered and on the dimension of the underlying Euclidean space.

We remark that the introduction of eigenvalues or of other quantities which may prove, in general, difficult to calculate has been deliberately avoided in the presentation.

Let $\Omega$ be an unbounded domain of $m$-dimensional Euclidean space $E^{m}$. We denote points of $E^{m}$ by $x=\left(x_{1}, \ldots, x_{m}\right)$ and differentiation with respect to $x_{i}$ by $D_{i}, i=1, \ldots, m$. For any subdomain $F$ of $\Omega$, we denote by $\|\cdot\|_{p}(F)$ the $L^{p}(F)$ norm and by $(\cdot, \cdot)$ the $L^{2}(F)$ inner product. If $F$ is obvious from the context we write $\|\cdot\|_{p}$ for $\|\cdot\|_{p}(F)$ etc. We consider the differential expression $L$ formally given in the standard multi-index notation by

$$
L u=(-1)^{n} \sum_{|\alpha|=|\beta|=n} D^{\alpha}\left(a_{\alpha \beta} D^{\beta} u\right)-a_{00} u, \quad a_{\alpha \beta}=a_{\beta \alpha} .
$$

The coefficients of $L$ are defined in $\Omega$, real, and sufficiently regular so that all

Received by the editors June 28, 1976.

AMS (MOS) subject classifications (1970). Primary 35B05, 35P15.

Key words and phrases. Elliptic equations, nonoscillation, conical domain, nodal domain. 
derivatives involved in $L$ exist and are at least continuous in $\Omega-R$ for some sphere $R$.

We further assume that $L$ is uniformly strongly elliptic in any bounded subdomain $F$ of $\Omega$. Consequently, it follows that by the usual means, see for example [2], $L$ can be used to define an operator, denoted by $\mathcal{L}$, whose domain is contained in the Sobolev space $H_{n}^{0}(F)$, formed by completing the space $C_{0}^{\infty}(F)$ in the Sobolev norm: $\|u\|^{2}=\sum_{|\alpha| \leqslant n} \int_{F}\left|D^{\alpha} u\right|^{2} d x$.

A bounded domain $F \subset \Omega$ is a nodal domain for $L$ iff there exists a nontrivial function $u \in H_{n}^{0}(F)$ such that $\mathcal{L} u=0$. $L$ is termed nonoscillatory in $\Omega$ iff there exists a sphere $R$, centered at the origin, such that $L$ has no nodal domains in $\Omega-R$.

With the symmetric expression $\left(a_{\alpha, \beta}\right)_{|\alpha|=|\beta|=n}$ it is possible to associate a symmetric matrix $\left(A_{i j}\right)_{i, j=1, \ldots, N}$ by the procedure of [1], [5]. Specifically let $\sigma$ be a bijection from the first $(n+m-1) ! / n !(m-1)$ ! integers to the set of $m$-tuples with sum $n$. We define $A_{i j}$ by $A_{i j}=a_{\sigma(i), \sigma(j)}$. Clearly the specific choice of $\sigma$ does not affect the smallest eigenvalue of $\left(A_{i j}\right)$.

We make one further restriction on the expression $L$. We assume that there exists an elliptic expression $l_{0}$, given by

$$
l_{0} u=(-1)^{n} \sum_{|\alpha|=|\beta|=n} D^{\alpha}\left(b_{\alpha \beta} D^{\beta} u\right),
$$

with constant coefficients and ellipticity constant $\mu_{0}$ such that the symmetric matrix associated with $\left(a_{\alpha \beta}(x)-b_{\alpha \beta}\right)$ is nonnegative definite for all $x \in \Omega-$ $R$. It then follows from the results of [1] that for $L$ to be nonoscillatory in $\Omega$ it is sufficient that the expression

$$
l_{1} u=(-1)^{n} \Delta^{n} u-a_{00} \mu_{0}^{-1} u
$$

be nonoscillatory in $\Omega$. In the sequel, we therefore obtain explicitly our results only for the expression $l_{1}$.

We also assume that $\Omega$ is sufficiently narrow so that for all $|x|$ sufficiently large it is contained in the cone $C_{\alpha}=\left\{x\left|x_{m}>\alpha\right| x \mid\right\}$ with $1>\alpha \geqslant 0$.

It is clear from the nature of the problem that it is sufficient to consider only subdomains of $\Omega$ which are in the complement of a sufficiently large sphere in $E^{m}$. Consequently, without loss of generality and without further mention we shall always assume that any function $\phi \in C_{0}^{\infty}(\Omega)$ considered in the sequel has support in $\{x|| x \mid>r\}$ where $r$ is chosen for convenience and sufficiently large.

We begin by stating the following well-known result, whose proof can be found, in particular, as an immediate consequence of the results of [7, p. 187].

Lemma 0 . Let $\phi \in C_{0}^{\infty}(\Omega), \gamma \leqslant 0, \omega \in C^{2}(\Omega)$ and $\omega$ positive in $\Omega$. Then the following inequality is valid:

$$
\int_{E^{m}}|x|^{\gamma} \sum_{i=1}^{m}\left(D_{i} \phi\right)^{2} \geqslant \int_{E^{m}} \phi^{2} \omega^{-1}\left\{\left[-\sum_{i=1}^{m} D_{i}\left[|x|^{\gamma} D_{i} \omega\right]\right]\right\} .
$$


We observe that the behaviour of $\omega$ on the boundary of $\Omega$ is immaterial to our considerations.

Corollary 0 . Let $\phi \in C_{0}^{\infty}(\Omega)$. Then the following inequality is valid:

$$
\int_{E^{m}}|x|^{\gamma} \sum_{i=1}^{m}\left(D_{i} \phi\right)^{2} \geqslant\left[\frac{(\gamma+m-2)^{2}}{4}+\frac{(m-1)}{1-\alpha}\right] \int_{E^{m}}|x|^{\gamma-2} \phi^{2}
$$

The proof of Corollary 0 follows immediately from the choice $\omega=$ $|x|^{-\delta}\left(x_{m}|x|^{-1}-\alpha\right)$, with $\delta=(m+\gamma-2) / 2$, in Lemma 0 . A better result could be obtained by the introduction of the eigenvalue of a singular Sturm-Liouville problem as was done in [3] for the case $\gamma=0$, but this eigenvalue would appear to be, in general, difficult to calculate explicitly.

LEMma 1. Let $\phi \in C_{0}^{\infty}(\Omega), \gamma \leqslant 0$. Then the following inequality holds:

$$
\begin{aligned}
\int_{E^{m}}|x|^{\gamma} & \sum_{i_{1}, \ldots, i_{n}=1}^{m}\left[D_{i_{1}} \cdots D_{i_{n}} \phi\right]^{2} \\
& \geqslant\left\{\prod_{j=0}^{n-1}\left[\frac{(\gamma+m-2-2 j)^{2}}{4}+\frac{(m-1)}{1-\alpha}\right]\right\} \int_{E^{m}}|x|^{-2 n+\gamma} \phi^{2}
\end{aligned}
$$

Proof. If $n=1$ then inequality (2) reduces to inequality (1). Assuming that inequality (2) is valid for $n=T$ we observe that

$$
\begin{aligned}
\int_{E^{m}}|x|^{\gamma} & \sum_{i_{1}, \ldots, i_{T+1}=1}^{m}\left[D_{i_{1}} \cdots D_{i_{T+1}} \phi\right]^{2} \\
= & \int_{E^{m}}|x|^{\gamma} \sum_{i_{2}, \ldots, i_{T+1}=1}^{m} \sum_{i_{1}=1}^{m}\left[D_{i_{1}}\left(D_{i_{2}} \cdots D_{i_{T+1}} \phi\right)\right]^{2} \\
\geqslant & {\left[\frac{(\gamma+m-2)^{2}}{4}+\frac{(m-1)}{1-\alpha}\right] \int_{E^{m}}|x|^{\gamma-2} \sum_{i_{2}, \ldots, i_{T+1}=1}^{m}\left(D_{i_{2}} \cdots D_{i_{T+1}} \phi\right)^{2} } \\
\geqslant & {\left[\frac{(\gamma+m-2)^{2}}{4}+\frac{(m-1)}{1-\alpha}\right] } \\
& \cdot\left\{\prod_{j=0}^{T-1}\left[\frac{(\gamma-4+m-2 j)^{2}}{4}+\frac{(m-1)}{1-\alpha}\right]\right\} \int_{E^{m}}|x|^{\gamma-2-2 T} \phi^{2} \\
= & \left\{\prod_{j=0}^{T}\left[\frac{(\gamma-2+m-2 j)^{2}}{4}+\frac{(m-1)}{1-\alpha}\right]\right\} \int_{E^{m}}|x|^{\gamma-2(T+1)} \phi^{2} .
\end{aligned}
$$


The statement of the lemma now follows by induction.

Combining the above results we can now state our first theorem:

Theorem 1. Assume that

$$
\underset{|x| \rightarrow \infty}{\lim \sup }\left[a_{00} \mu_{0}^{-1}|x|^{+2 n}\right]<\prod_{j=0}^{n-1}\left[\frac{(m-2-2 j)^{2}}{4}+\frac{(m-1)}{1-\alpha}\right] .
$$

Then the expression $l_{1}$ is nonoscillatory in $\Omega$.

Proof. We first observe that for any function $\phi \in C_{0}^{\infty}(\Omega)$ we have as an immediate consequence of Green's formula the identity:

$$
\int_{E^{m}} \phi(-1)^{n} \Delta^{n} \phi=\int_{E^{m}} \sum_{i_{1}, \ldots, i_{n}=1}^{m}\left[D_{i_{1}} \cdots D_{i_{n}} \phi\right]^{2} .
$$

If we assume that $l_{1}$ is oscillatory then given any sphere $R$ there exist a bounded domain $F \subset \Omega-R$ and a nontrivial function $u \in H_{n}^{0}(F)$ such that

$$
(-1)^{n}\left(u, \Delta^{n} u\right)-\left(a_{00} \mu_{0}^{-1} u, u\right) \leqslant 0 .
$$

Furthermore, by a simple limit argument we see that inequality (2) is also valid for $u$. Estimating the first inner product of (4) by means of (2) leads to the desired contradiction.

We note that the constant on the right-hand side of (3) is positive, regardless of the values of $m$ and $n$. This is considerably different from the results obtained for the case where $\Omega$ is the complement of a sphere in $E^{m}$, where for some values of $n$ and $m$, a result such as (3) is known to be false. As an example of such a case it is sufficient to take the case $n=1, m=2$ where the best result is known to be [1]

$$
\underset{|x| \rightarrow \infty}{\lim \sup }\left[a_{00} \mu_{0}^{-1}|x|^{+2}(\ln |x|)^{2}\right]<\frac{1}{4} \text {. }
$$

We also observe that as a consequence of Theorem 1 it follows that if $a_{00}$ is defined in the complement of a sphere and if, for some $\tau$,

$$
\underset{|x| \rightarrow \infty}{\lim \sup }\left[a_{00} \mu_{0}^{-1}|x|^{2 n}\right]=\tau<+\infty
$$

then $l_{1}$ is nonoscillatory in the cones $C_{\alpha}$ where $\alpha$ is any number which makes the right-hand side of (3) greater than $\tau$. To illustrate this remark we consider the case of the operation $l_{2} u=\Delta^{2} u-k^{2}|x|^{-4} u=0$ in $E^{3}$, with $k$ large. In this case we find that $l_{2}$ is nonoscillatory in any cone $C_{\alpha}$ where

$$
1>\alpha>(4 k-9)(4 k-1)^{-1} \text {. }
$$

We also compare the result of Theorem 1 with the known result [1] for the case where $m>2 n$ and $\Omega$ is the complement of a sphere. In such a case the nonoscillation of $l_{1}$ is implied by the condition 


$$
\limsup _{|x| \rightarrow \infty}\left[a_{00} \mu_{0}^{-1}|x|^{2 n}\right]<\prod_{j=0}^{n-1} \frac{(m+4 j-2 n)^{2}}{4} .
$$

Condition (3) is clearly better than condition (5) when the cone is sufficiently narrow, that is, for $\alpha$ near 1 .

In conclusion we remark that extended Sobolev estimates can be used to obtain integral nonoscillation criteria similar to those of [1].

THEOREM 2. Assume that $m>2 n$ and that for some $\varepsilon, 0<\varepsilon<1$, there exists a sphere $R$ such that

$$
\left[\varepsilon|x|^{-2 n} \mu_{0} \prod_{j=0}^{n-1}\left\{\frac{(m-2-2 j)^{2}}{4}+\frac{(m-1)}{1-\alpha}\right\}-a_{00}\right]_{-} \in L^{m / 2 n}(\Omega-R) .
$$

Then the expression $l_{1}$ is nonoscillatory in $\Omega$.

Proof. Set

$$
v=\mu_{0} \prod_{j=0}^{n-1}\left\{\frac{(m-2-2 j)^{2}}{4}+\frac{(m-1)}{1-\alpha}\right\} .
$$

Proceeding as in [1] we find that if $l_{1}$ is oscillatory then for any sphere $R_{1}$ there exists a bounded domain $F \subset \Omega-R_{1}$ and function $u \in H_{n}^{0}(F)$ such that

$$
\left(u,(-1)^{n} \Delta^{n} u\right)-\left(a_{00} \mu_{0}^{-1} u, u\right) \leqslant 0 .
$$

Employing Lemma 1, with $\gamma=0$, we find

$$
\left(u,(-1)^{n} \Delta^{n} u\right) \geqslant(1-\varepsilon)\left(u,(-1)^{n} \Delta^{n} u\right)+v \mu_{0}^{-1} \varepsilon\left(|x|^{-2 n} u, u\right) .
$$

Substituting this result into (6) gives

$$
\left(u,(-1)^{n} \Delta^{n} u\right)-\frac{\mu_{0}^{-1}}{1-\varepsilon}\left(\left(|x|^{-2 n} v \varepsilon-a_{00}\right)_{-} u, u\right) \leqslant 0 .
$$

We observe that the second inner product in (7) can be estimated as was done in [1] by means of Hölder's inequality and generalized Sobolev estimates [2, $\mathrm{p}$. 24], so that for some constant $C$, inequality (7) becomes

$$
\text { (4) }\left(u,(-1)^{n} \Delta^{n} u\right)\left[1-\frac{C}{1-\varepsilon}\left\|\left(\varepsilon v|x|^{-2 n}-a_{00}\right)_{-}\right\|_{m / 2 n}\left(\Omega-R_{1}\right)\right] \leqslant 0 \text {. }
$$

Choosing $R_{1}$ sufficiently large gives the desired contradiction.

\section{REFERENCES}

1. W. Allegretto, Nonoscillation theory of elliptic equations of order $2 n$, Pacific J. Math. 64 (1976), 1-16.

2. A. Friedman, Partial differential equations, Holt, Rinehart and Winston, New York, 1969. 
3. V. B. Headley and C. A. Swanson, Oscillation criteria for elliptic equations, Pacific J. Math. 27 (1968), 501-506. MR 38 \#4797.

4. K. Kreith, Oscillation theory, Lecture Notes in Math., Vol. 324, Springer-Verlag, Berlin and New York, 1973.

5. E. S. Noussair, Oscillation theory of elliptic equations of order $2 m$, J. Differential Equations 10 (1971), 100-111. MR 43 \#6564.

6. E. Noussair and N. Yoshida, Nonoscillation criteria for elliptic equations of order $2 m$ (submitted).

7. C. A. Swanson, Comparison and oscillation theory of linear differential equations, Academic Press, New York, 1968.

Department of Mathematics, University of Alberta, Edmonton, Alberta, Canada 